\title{
Switching and stopping antidepressants
}

\section{Nicholas Keks \\ Director \\ Adjunct professor ${ }^{2}$ \\ Judy Hope \\ Deputy director \\ Senior lecturer ${ }^{2}$ \\ Simone Keogh \\ Psychiatrist and senior fellow? \\ ${ }^{1}$ Centre for Mental Health \\ Education and Research \\ Delmont Private Hospital \\ 2 Monash University \\ Melbourne}

\section{Keywords}

antidepressant, drug

interaction, drug

withdrawal, serotonin syndrome

Aust Prescr 2016;39:76-83 http://dx.doi.org/10.18773/ austprescr.2016.039

\section{SUMMARY}

Switching from one antidepressant to another is frequently indicated due to an inadequate treatment response or unacceptable adverse effects. All antidepressant switches must be carried out cautiously and under close observation.

Conservative switching strategies involve gradually tapering the first antidepressant followed by an adequate washout period before the new antidepressant is started. This can take a long time and include periods of no treatment with the risk of potentially life-threatening exacerbations of illness.

Clinical expertise is needed for more rapid or cross-taper switching as drug toxicity, including serotonin syndrome, may result from inappropriate co-administration of antidepressants. Some antidepressants must not be combined.

Antidepressants can cause withdrawal syndromes if discontinued abruptly after prolonged use. Relapse and exacerbation of depression can also occur. Gradual dose reduction over days to weeks reduces the risk and severity of complications.

\section{Introduction}

Antidepressant drugs are indicated for the treatment of depression, anxiety disorders (including panic and social phobia), obsessive compulsive disorder and post-traumatic stress disorder. There are over 20 antidepressants currently available in Australia. These can be divided into 13 clinically relevant groups, which differ substantially in their pharmacodynamic and pharmacokinetic characteristics.

Up to two-thirds of patients with major depression fail to respond to their first antidepressant drug. After assuring correct diagnosis, optimal dose, duration and adherence to treatment, a change of antidepressant drug is indicated.' A patient is unlikely to respond if there has been no improvement after three to four weeks on an adequate dose of antidepressant. ${ }^{2}$ About a quarter of patients switched to a second antidepressant can be expected to achieve remission. ${ }^{3}$ There is no evidence that switching between classes of antidepressants is more effective than switching within a class. ${ }^{4}$ Unacceptable adverse effects from antidepressants, such as sexual dysfunction and weight gain, may also necessitate a change of therapy. ${ }^{5}$ Switching from one antidepressant to another is a common clinical challenge.

Withdrawal of an antidepressant is also indicated after an episode of depression has been adequately treated - usually six to nine months after recovery from a single episode. Serious physical illness, pregnancy and surgery may also be reasons for stopping antidepressant therapy. Up to a third of patients stop antidepressants soon after starting and many more only partially adhere to treatment. ${ }^{6}$

\section{Withdrawing antidepressants}

If used for longer than six weeks, all antidepressants have the potential to cause withdrawal syndromes if they are stopped or rapidly reduced (with the possible exception of agomelatine). As a result many patients believe that antidepressants are addictive. This is not the case as abusive and compulsive use, tolerance and drug seeking do not occur with antidepressant drugs. Withdrawal syndromes occur with many drugs (such as corticosteroids) when used long term.

The usual recommended period for antidepressant dose reduction is a minimum of four weeks. ${ }^{2}$ However, abrupt cessation may at times be unavoidable on clinical grounds. The time frame for dose reduction also depends on individual risk for withdrawal symptoms, patient preference and experience during withdrawal, and drug characteristics such as half-life (Table 1).

Previous withdrawal symptoms and anxiety when starting antidepressant treatment are predictors of future discontinuation problems. Some patients experience little discomfort despite abrupt cessation, while others are severely affected. In a minority, withdrawal symptoms are not diminished by extending the duration of dose taper. These patients may prefer rapid cessation and a briefer withdrawal period. Many will not experience symptoms in the early part of withdrawal (which could proceed more rapidly) but develop severe symptoms in the 
later stages (when dose reduction may need to be more gradual).

\section{Withdrawal symptoms}

Withdrawal symptoms generally begin within hours to days of dose reduction, depending on the characteristics of the particular drug. ${ }^{7}$ Withdrawing selective serotonin reuptake inhibitors (SSRIs) and serotonin noradrenaline reuptake inhibitors (SNRIs) tends to cause flu-like symptoms, nausea, lethargy, dizziness, ataxia, 'electric shock' sensations, anxiety, irritability, insomnia and vivid dreams. The symptoms can be extremely disabling for some patients.

Venlafaxine is associated with the most severe withdrawal effects. Paroxetine is also troublesome while fluoxetine rarely causes withdrawal symptoms (especially if the dose is under $40 \mathrm{mg}$ ) due to the long half-life of the parent drug and its active metabolite (about 7 days). Withdrawal of tricyclic antidepressants can cause nausea, headache, abdominal pain, diarrhoea, lethargy, anxiety, insomnia and vivid dreams. It is unlikely that withdrawal symptoms will occur after cessation of low-dose tricyclics used in pain treatment. Withdrawing irreversible monoamine oxidase inhibitors such as tranylcypromine is particularly troublesome. It often causes agitation, irritability, mood disorders, dreams, cognitive impairment and occasionally psychosis and delirium.

\section{Relapse and exacerbation}

Stopping antidepressants can also result in relapse or exacerbation of the psychiatric illness. Relapse of depressive symptoms (including suicidal ideation and self-harm) and recurrence of panic attacks and severe anxiety can all occur with dose reduction and cessation. Such exacerbations can cause lifethreatening behaviours in high-risk patients, and antidepressant withdrawal must be a carefully considered decision made by the well-informed patient, often their family, and the prescriber. Avoid stopping an antidepressant abruptly - withdrawal over weeks to months (if possible) reduces the risk of relapse. ${ }^{2}$

\section{Switching strategies}

A number of strategies are available for switching between antidepressants (Table 2). ${ }^{6,8}$ Close clinical observation and caution is required with all approaches, as some patients may respond idiosyncratically and serious complications can occur. Individual patient factors and illness factors may require considerable modification of a switching strategy.

The most conservative strategy, with the lowest risk of drug interactions, is to gradually taper the dose of the first antidepressant to minimise withdrawal symptoms then start a washout period equivalent to five halflives of the drug (Table 1). This does not apply to irreversible monoamine oxidase inhibitors where a specified long period of washout is mandatory (see Table 3). Five half-lives equates to about five days for most SSRIs except fluoxetine, which can still be significantly active five or more weeks after cessation. The second antidepressant is then introduced according to the starting dose recommendations.

\section{Table 1 Approximate half-lives of antidepressants}

\begin{tabular}{|c|c|}
\hline Antidepressant & $\begin{array}{l}\text { Approximate half-life } \\
\text { (days) }\end{array}$ \\
\hline citalopram & 1.5 \\
\hline escitalopram & 1.5 \\
\hline paroxetine & 1.0 \\
\hline sertraline & $1.1-1.3$ \\
\hline fluoxetine & $4-16^{*}$ \\
\hline fluvoxamine & 0.6 \\
\hline vortioxetine & $2.4-2.8$ \\
\hline agomelatine & $0.04-0.08$ \\
\hline desvenlafaxine & 0.4 \\
\hline duloxetine & 0.5 \\
\hline venlafaxine & $0.6^{\dagger}$ \\
\hline mianserin & $0.9-2.5$ \\
\hline mirtazepine & $0.8-1.6 \ddagger$ \\
\hline reboxetine & 0.5 \\
\hline amitriptyline & $0.2-1.9$ \\
\hline imipramine & $0.2-1.3$ \\
\hline nortriptyline & $0.8-2.3$ \\
\hline doxepin & $0.4-1.0$ \\
\hline dothiepin & 2.1 \\
\hline trimipramine & $0.6-1.6$ \\
\hline clomipramine & $0.6-2.5$ \\
\hline moclobemide & 0.08 \\
\hline phenelzine & see below $§$ \\
\hline tranylcypromine & see below ${ }^{\S}$ \\
\hline
\end{tabular}

* fluoxetine plus active metabolite norfluoxetine

+ venlafaxine plus active metabolite desvenlafaxine

$\ddagger$ a longer half-life (up to 65 hours) has occasionally been recorded and a shorter half-life is sometimes seen in young men

$\S$ biological activity persists for 14-21 days 
The dose is usually tapered over four weeks, similar to the minimum period required for antidepressant discontinuation. However, the time frame may need to be modified depending on patient factors.

As the conservative switch can take quite a long time and usually includes at least several days where the patient is not on an antidepressant, a compromise strategy is the moderate switch. Here the washout period can generally be shortened to about two days. The risk of drug interactions is increased with this approach, but is still quite low. The conservative and moderate switch techniques are both suitable for general practice.

Direct and cross-taper switch methods can also be used but considerable expertise is necessary (Table 2). Some patients will require admission to hospital. A direct switch - one drug is stopped and another drug is commenced the next day at the usual therapeutic dose - can be used when switching between some SSRIs, SNRIs and tricyclic antidepressants. However, there will be a considerable risk of withdrawal symptoms and drug interactions. A cross-taper strategy, where the first antidepressant dose is reduced while the second antidepressant is introduced at a low dose and gradually increased, can be done safely with only some antidepressants (Table 3).

\section{Switching between specific antidepressants}

Table 3 lists generalised guidelines for switching patients from one antidepressant to another. ${ }^{2,8-10}$ The recommendations are applicable to any switching strategy. Circumstances where only a conservative strategy can be used are identified. Table 3 also states when antidepressants should not be co-administered or tapered at the same time.

\section{Serotonin syndrome}

As many antidepressants have serotonergic activity, serotonin syndrome can occur during antidepressant switching. While the syndrome may cause mild

\section{Table 2 Techniques for switching from one antidepressant to another ${ }^{6}$}

\begin{tabular}{|c|c|}
\hline Method & Comment \\
\hline $\begin{array}{l}\text { Conservative switch: } \\
\text { - the first antidepressant is gradually reduced and stopped } \\
\text { - there follows a drug-free washout interval of five half-lives } \\
\text { of the first antidepressant } \\
\text { - the new antidepressant is started according to its dose } \\
\text { recommendation }\end{array}$ & $\begin{array}{l}\text { Most appropriate for general practice. The risk of drug } \\
\text { interactions is very low but discontinuation symptoms } \\
\text { may occur. }\end{array}$ \\
\hline $\begin{array}{l}\text { Moderate switch: } \\
\text { - the first antidepressant is gradually reduced and stopped } \\
\text { - there follows a drug-free washout interval of } 2-4 \text { days } \\
\text { - the new antidepressant is started at a low dose }\end{array}$ & $\begin{array}{l}\text { Also recommended for use in general practice. The risk } \\
\text { of drug interactions is low but discontinuation symptoms } \\
\text { may occur. }\end{array}$ \\
\hline $\begin{array}{l}\text { Direct switch: } \\
\text { - the first antidepressant is stopped } \\
\text { - the second antidepressant is started the next day at the } \\
\text { usual therapeutic dose }\end{array}$ & $\begin{array}{l}\text { Quick and simple but discontinuation symptoms are likely } \\
\text { depending on the second antidepressant. The risk of } \\
\text { drug interactions is substantial, depending on the second } \\
\text { antidepressant. Method requires clinical expertise and is } \\
\text { only feasible in selected instances, such as swapping from } \\
\text { one short half-life SSRI to another. }\end{array}$ \\
\hline $\begin{array}{l}\text { Cross-taper switch: } \\
\text { - the first antidepressant is gradually reduced and stopped } \\
\text { - the second antidepressant is introduced at a low dose at } \\
\text { some stage during the reduction of the first antidepressant, } \\
\text { so that the patient is taking both antidepressants } \\
\text { simultaneously } \\
\text { - the dose of the second antidepressant is increased to } \\
\text { the therapeutic dose when the first antidepressant has } \\
\text { been stopped }\end{array}$ & $\begin{array}{l}\text { Frequently used for patients with high risk from illness } \\
\text { relapse but there is risk of drug interactions and increased } \\
\text { adverse effects from combined medications. Only feasible } \\
\text { in selected instances. Requires clinical expertise. }\end{array}$ \\
\hline
\end{tabular}

Note: Above strategies do not apply to monamine oxidase inhibitors, for which strict recommendations must be followed (Table 3)

SSRI selective serotonin reuptake inhibitor

Adapted from reference 6 
symptoms such as nervousness, agitation, tremor, diaphoresis, shivering, mydriasis, hyperreflexia and diarrhoea, in more severe cases tachycardia, hyperthermia, hypertension, myoclonus, muscular rigidity and delirium can occur. Convulsions, organ system failure and death may follow. Prevention through minimising interactions between potent serotonergic drugs is critical. ${ }^{11}$

The only significant interaction for agomelatine is with fluvoxamine (Table 3). Vortioxetine (an SSRI with possible other serotonergic effects) can interact with a variety of antidepressants. Caution is required for switching and the prescriber should consult relevant drug information before proceeding. The same caution applies to duloxetine (Table 3 ).

Fluoxetine is a particular challenge for switching because of its long half-life. Serotonin syndrome can occur if clomipramine, fluvoxamine or monoamine oxidase inhibitors are introduced before an adequate washout of fluoxetine, which can take five or more weeks. Tricyclic antidepressants can be introduced at a low dose after fluoxetine withdrawal. However, the low dose needs to be continued for several weeks to avoid cardiotoxic plasma concentrations of tricyclic antidepressant due to inhibition of tricyclic antidepressant metabolism by fluoxetine. Early signs of tricyclic antidepressant toxicity include drowsiness, tachycardia and postural hypotension.

When changing from irreversible monoamine oxidase inhibitors (phenelzine and tranylcypromine) to all other antidepressants, with the possible exception of agomelatine, an adequate washout of two to three weeks is mandatory.

\section{Conclusion}

Switching antidepressants involves drug cessation, which may cause withdrawal symptoms and relapse or exacerbation of the psychiatric illness. Gradual antidepressant withdrawal reduces the risk of complications. If the washout period is not long enough (defined by half-life of the drug), introducing a new antidepressant can cause drug interactions leading to toxicity, particularly serotonin syndrome. Switching from one antidepressant to another requires careful observation and caution. $<$

Conflict of interest: none declared

\section{REFERENCES}

1. Little A. Treatment-resistant depression. Am Fam Physician 2009;80:167-72.

2. Taylor D, Paton C, Kapur S, editors. The Maudsley prescribing guidelines in psychiatry. 11th ed. London: Wiley Blackwell; 2015.

3. Rush AJ, Trivedi MH, Wisniewski SR, Nierenberg AA Stewart JW, Warden D, et al. Acute and longer-term outcomes in depressed outpatients requiring one or several treatment steps: a STAR*D report. Am J Psychiatry 2006:163:1905-17. http://dx.doi.org/10.1176/ajp.2006.163.11.1905

4. Souery D, Serretti A, Calati R, Oswald P, Massat I, Konstantinidis A, et al. Switching antidepressant class does not improve response or remission in treatment-resistant depression. J Clin Psychopharmacol 2011;31:512-6. http://dx.doi.org/10.1097/JCP.0b013e3182228619

5. Keks NA, Hope J, Culhane C. Management of antidepressant induced sexual dysfunction. Australas Psychiatry 2014:22:525-8. http://dx.doi.org/10.1177/1039856214556323
6. Jefferson JW. Strategies for switching antidepressants to achieve maximum efficacy. J Clin Psychiatry 2008;69 Suppl E1:14-8.

7. Schweitzer I, Maguire K. Stopping antidepressants Aust Prescr 2001;24:13-5. http://dx.doi.org/10.18773/ austprescr.2001.008

8. Luft B. Antidepressant switching strategies. Graylands Hospital Drug Bulletin 2013;20:1-4

9. Psychotropic Expert Group. Therapeutic Guidelines: psychotropic. Version 7. Melbourne: Therapeutic Guidelines Limited; 2013.

10. Procyshyn RM, Bezchlibnyk-Butler KZ, Jeffries JJ, editors. Clinical handbook of psychotropic drugs. 21st ed. Boston: Hogrefe Publishing; 2015.

11. Buckley NA, Dawson AH, Isbister GK. Serotonin syndrome. BMJ 2014;348:g1626. http://dx.doi.org/10.1136/bmj.g1626

\section{FURTHER READING}

Fava GA, Gatti A, Belaise C, Guidi J, Offidani E. Withdrawal symptoms after selective serotonin reuptake inhibitor

discontinuation: a systematic review. Psychother Psychosom

2015;84:72-81. http://dx.doi.org/10.1159/000370338 


\section{Table 3 Guidelines for switching between specific antidepressants ${ }^{2,8-10}$}

\begin{tabular}{|c|c|c|c|c|c|c|}
\hline 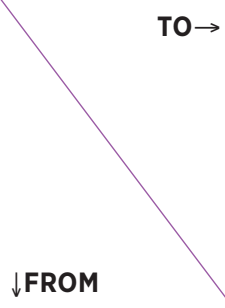 & $\begin{array}{l}\text { citalopram } \\
\text { escitalopram } \\
\text { paroxetine } \\
\text { sertraline } \\
\text { (SSRIs) }\end{array}$ & fluoxetine & fluvoxamine & vortioxetine & agomelatine & $\begin{array}{l}\text { desvenlafaxine } \\
\text { duloxetine } \\
\text { venlafaxine } \\
\text { (SNRIs) }\end{array}$ \\
\hline $\begin{array}{l}\text { citalopram } \\
\text { escitalopram } \\
\text { paroxetine } \\
\text { sertraline } \\
\text { (SSRIs) }\end{array}$ & $\begin{array}{l}\text { taper drug, start } \\
\text { alternative SSRI } \\
\text { at low dose* }\end{array}$ & $\begin{array}{l}\text { taper and stop } \\
\text { drug, then start } \\
\text { fluoxetine at } \\
10 \mathrm{mg}^{\S}\end{array}$ & $\begin{array}{l}\text { taper and stop } \\
\text { drug, then start } \\
\text { fluvoxamine at } \\
50 \mathrm{mg}^{\S}\end{array}$ & $\begin{array}{l}\text { taper drug, start } \\
\text { vortioxetine at } \\
5 \mathrm{mg}^{*}\end{array}$ & $\begin{array}{l}\text { taper drug, start } \\
\text { agomelatine* }\end{array}$ & $\begin{array}{l}\text { taper drug, then } \\
\text { start SNRI at low } \\
\text { dose }^{*}\end{array}$ \\
\hline fluoxetine & $\begin{array}{l}\text { stop fluoxetine } \\
\text { (or taper if dose } \\
>40 \mathrm{mg} / \mathrm{day} \text { ), } \\
\text { wait } 7 \text { days for } \\
\text { washout, then } \\
\text { start above SSRI } \\
\text { at low dose }\end{array}$ & & $\begin{array}{l}\text { stop fluoxetine } \\
\text { (or taper if dose } \\
>40 \mathrm{mg} / \text { day), } \\
\text { wait } 14 \text { days for } \\
\text { washout, then } \\
\text { start fluvoxamine } \\
\text { at } 50 \mathrm{mg}^{\dagger \S}\end{array}$ & $\begin{array}{l}\text { stop fluoxetine } \\
\text { (or taper if dose } \\
>40 \mathrm{mg} / \text { day), } \\
\text { wait } 7 \text { days for } \\
\text { washout, then } \\
\text { start vortioxetine } \\
\text { at } 5 \mathrm{mg}^{\dagger \S}\end{array}$ & $\begin{array}{l}\text { stop fluoxetine } \\
\text { (or taper if dose } \\
>40 \mathrm{mg} / \text { day), } \\
\text { start agomelatine }\end{array}$ & $\begin{array}{l}\text { taper and stop } \\
\text { fluoxetine, } \\
\text { wait } 7 \text { days for } \\
\text { washout, then } \\
\text { start SNRI at low } \\
\text { dose }^{+\S}\end{array}$ \\
\hline fluvoxamine & $\begin{array}{l}\text { taper and stop } \\
\text { fluvoxamine, then } \\
\text { start above SSRI } \\
\text { at low dose }\end{array}$ & $\begin{array}{l}\text { taper and stop } \\
\text { fluvoxamine, then } \\
\text { start fluoxetine at } \\
10 \mathrm{mg}^{\S}\end{array}$ & & $\begin{array}{l}\text { taper and stop } \\
\text { fluvoxamine, start } \\
\text { vortioxetine at } \\
5 \mathrm{mg}^{\S}\end{array}$ & $\begin{array}{l}\text { taper and stop } \\
\text { fluvoxamine, } \\
\text { wait } 7 \text { days } \\
\text { for washout, } \\
\text { then start } \\
\text { agomelatine }^{\S}\end{array}$ & $\begin{array}{l}\text { taper and stop } \\
\text { fluvoxamine, then } \\
\text { start SNRI at low } \\
\text { dose }^{\S}\end{array}$ \\
\hline vortioxetine & $\begin{array}{l}\text { taper } \\
\text { vortioxetine, start } \\
\text { above SSRI at low } \\
\text { dose* }\end{array}$ & $\begin{array}{l}\text { taper and stop } \\
\text { vortioxetine, } \\
\text { start fluoxetine at } \\
10 \mathrm{mg}^{\S}\end{array}$ & $\begin{array}{l}\text { taper and stop } \\
\text { vortioxetine, start } \\
\text { fluvoxamine at } \\
50 \mathrm{mg}^{\S}\end{array}$ & & $\begin{array}{l}\text { taper } \\
\text { vortioxetine, start } \\
\text { agomelatine at } \\
25 \mathrm{mg}^{*}\end{array}$ & $\begin{array}{l}\text { taper } \\
\text { vortioxetine, start } \\
\text { SNRI at low dose* }\end{array}$ \\
\hline agomelatine & $\begin{array}{l}\text { stop agomelatine, } \\
\text { then start above } \\
\text { SSRI }\end{array}$ & $\begin{array}{l}\text { stop agomelatine, } \\
\text { then start } \\
\text { fluoxetine }\end{array}$ & $\begin{array}{l}\text { stop agomelatine, } \\
\text { then start } \\
\text { fluvoxamine* }\end{array}$ & $\begin{array}{l}\text { stop agomelatine, } \\
\text { then start } \\
\text { vortioxetine }\end{array}$ & & $\begin{array}{l}\text { stop agomelatine, } \\
\text { then start SNRI }\end{array}$ \\
\hline $\begin{array}{l}\text { desvenlafaxine } \\
\text { duloxetine } \\
\text { venlafaxine } \\
\text { (SNRIs) }\end{array}$ & $\begin{array}{l}\text { taper SNRI, start } \\
\text { above SSRI at low } \\
\text { dose* }\end{array}$ & $\begin{array}{l}\text { taper and stop } \\
\text { SNRI, start } \\
\text { fluoxetine at } \\
10 \mathrm{mg}^{\S}\end{array}$ & $\begin{array}{l}\text { taper and stop } \\
\text { SNRI, start } \\
\text { fluvoxamine at } \\
50 \mathrm{mg}^{\S}\end{array}$ & $\begin{array}{l}\text { taper SNRI, start } \\
\text { vortioxetine at } \\
5 \mathrm{mg}^{*}\end{array}$ & $\begin{array}{l}\text { taper SNRI, start } \\
\text { agomelatine* }\end{array}$ & $\begin{array}{l}\text { taper SNRI, start } \\
\text { alternative SNRI } \\
\text { at low dose* }\end{array}$ \\
\hline $\begin{array}{l}\text { mianserin } \\
\text { mirtazepine }\end{array}$ & $\begin{array}{l}\text { taper drug, start } \\
\text { above SSRI* }\end{array}$ & $\begin{array}{l}\text { taper drug, start } \\
\text { fluoxetine* }\end{array}$ & $\begin{array}{l}\text { taper drug, start } \\
\text { fluvoxamine* }\end{array}$ & $\begin{array}{l}\text { taper drug, start } \\
\text { vortioxetine* }\end{array}$ & $\begin{array}{l}\text { taper drug, start } \\
\text { agomelatine* }\end{array}$ & $\begin{array}{l}\text { taper drug, start } \\
\text { SNRI* }\end{array}$ \\
\hline reboxetine & $\begin{array}{l}\text { taper reboxetine, } \\
\text { start above SSRI* }\end{array}$ & $\begin{array}{l}\text { taper reboxetine, } \\
\text { start fluoxetine* }\end{array}$ & $\begin{array}{l}\text { taper reboxetine, } \\
\text { start fluvoxamine } \\
\text { at } 50 \mathrm{mg}^{*}\end{array}$ & $\begin{array}{l}\text { taper reboxetine, } \\
\text { start vortioxetine } \\
\text { at } 5 \mathrm{mg}^{*}\end{array}$ & $\begin{array}{l}\text { taper reboxetine, } \\
\text { start agomelatine* }\end{array}$ & $\begin{array}{l}\text { taper reboxetine, } \\
\text { start SNRI at low } \\
\text { dose* }^{*}\end{array}$ \\
\hline
\end{tabular}




\section{mianserin}

mirtazapine reboxetine

amitriptyline
imipramine
nortriptyline
doxepin
dothiepin
trimipramine
(TCAs)

taper drug, then start taper drug, start above drug at low reboxetine* taper SSRI, start above drug at

$25 \mathrm{mg})^{*}$

\section{clomipramine}

moclobemide

phenelzine

tranylcypromine

(MAOIs) dose* low dose (usually taper and stop

drug, then start

clomipramine at

$25 \mathrm{mg}^{\S}$ taper and stop drug

for 7 days washout

before starting

moclobemide at

low dose s $^{-}$ taper and stop drug for 7 days washout before starting MAOI at low dose s $^{\text {s }}$ stop fluoxetine

(or taper if dose

$>40$ mg/day), wait

14 days for washout, then start above drug at $25 \mathrm{mg}$ and continue low dose for further 3 weeks

\begin{abstract}
$$
\begin{aligned}
& \text { taper fluvoxamine, } \\
& \text { start reboxetine at }
\end{aligned}
$$
$4 \mathrm{mg}^{*}$

$>40$ mg/day), start reboxetine at $4 \mathrm{mg}$
\end{abstract}

\begin{abstract}
taper fluvoxamine, start above drug at $25 \mathrm{mg}^{*}$
\end{abstract}

stop fluoxetine (or taper if dose $>40$ mg/day), wait 14 days for washout, then start clomipramine at $25 \mathrm{mg}$ and continue this dose for further 3 weekst

taper and stop start above drug at low dose§

taper and stop fluvoxamine, start clomipramine at $25 \mathrm{mg}^{\S}$

$\begin{array}{ll}\begin{array}{ll}\text { stop fluoxetine } \\ \text { (or taper if dose }\end{array} & \text { stop fluoxetine } \\ >40 \mathrm{mg} / \text { day), then } & \text { (or taper if dose } \\ \text { wait 5-6 weeks } & \text { wait 5-6 weeks } \\ \text { for washout } & \text { for washout } \\ \text { before cautiously } & \text { before cautiously } \\ \text { commencing low- } & \text { commencing low- } \\ \text { dose moclobemide } & \text { dose MAOI }\end{array}$

taper and stop taper and stop fluvoxamine, wait fluvoxamine, wait 7 days for washout 7 days for washout before cautiously before cautiously commencing low- commencing lowdose moclobemide ${ }^{\S}$ dose MAOI $^{\S}$

taper and stop taper and stop vortioxetine for vortioxetine for 14 days washout $\quad 21$ days washout before starting before starting moclobemide at MAOI at low dose low dose cautiously $^{\S}$ start above drug at low dose (usually $25 \mathrm{mg})^{*}$

taper and stop vortioxetine, start clomipramine at $25 \mathrm{mg}^{\S}$ low dose* taper vortioxetine
start reboxetine* stop agomelatine, then start reboxetine then start above drug

\section{taper SNRI, start} above drug at low dose*

\section{taper SNRI, start reboxetine at $4 \mathrm{mg}^{*}$ taper SNRI, start above drug at 25 mg*}

stop agomelatine, then start above drug at low dose (usually $25 \mathrm{mg}$ )*

\section{taper drug, start reboxetine at $4 \mathrm{mg}^{*}$}

\begin{abstract}
taper drug, start above drug at 25 mg*
\end{abstract}

\begin{abstract}
taper drug, start clomipramine at 25 mg*
\end{abstract}

stop agomelatine, then start clomipramine taper SNRI, start clomipramine at 25 mg*

\begin{abstract}
taper drug, start
drug above at low dose*
\end{abstract} dose

$25 \mathrm{mg}^{*}$


Table 3 Guidelines for switching between specific antidepressants ${ }^{2,8-10}$ (continued)

\begin{tabular}{|c|c|c|c|c|c|c|}
\hline TO $\rightarrow$ & $\begin{array}{l}\text { citalopram } \\
\text { escitalopram } \\
\text { paroxetine } \\
\text { sertraline } \\
\text { (SSRIs) }\end{array}$ & fluoxetine & fluvoxamine & vortioxetine & agomelatine & $\begin{array}{l}\text { desvenlafaxine } \\
\text { duloxetine } \\
\text { venlafaxine } \\
\text { (SNRIs) }\end{array}$ \\
\hline $\begin{array}{l}\text { amitriptyline } \\
\text { imipramine } \\
\text { nortriptyline } \\
\text { doxepin } \\
\text { dothiepin } \\
\text { trimipramine } \\
\text { (TCAs) }\end{array}$ & $\begin{array}{l}\text { taper first drug } \\
\text { and start above } \\
\text { drug at low dose* }\end{array}$ & $\begin{array}{l}\text { taper and } \\
\text { stop first drug } \\
\text { before starting } \\
\text { fluoxetine }^{\S}\end{array}$ & $\begin{array}{l}\text { taper drug, start } \\
\text { fluvoxamine at } \\
50 \mathrm{mg}^{*}\end{array}$ & $\begin{array}{l}\text { taper drug, start } \\
\text { vortioxetine at } \\
5 \mathrm{mg}^{*}\end{array}$ & $\begin{array}{l}\text { taper drug, start } \\
\text { agomelatine* }\end{array}$ & $\begin{array}{l}\text { taper drug, start } \\
\text { SNRI at low dose* }\end{array}$ \\
\hline clomipramine & $\begin{array}{l}\text { taper and stop } \\
\text { clomipramine, } \\
\text { then start above } \\
\text { SSRI at low dose }\end{array}$ & $\begin{array}{l}\text { taper and stop } \\
\text { clomipramine, } \\
\text { then start } \\
\text { fluoxetine at } \\
10 \mathrm{mg}^{\S}\end{array}$ & $\begin{array}{l}\text { taper and stop } \\
\text { clomipramine, } \\
\text { then start } \\
\text { fluvoxamine at } \\
50 \mathrm{mg}^{\S}\end{array}$ & $\begin{array}{l}\text { taper and stop } \\
\text { clomipramine, } \\
\text { then start } \\
\text { vortioxetine at } \\
5 \mathrm{mg}^{\S}\end{array}$ & $\begin{array}{l}\text { taper } \\
\text { clomipramine, } \\
\text { start } \\
\text { agomelatine* }\end{array}$ & $\begin{array}{l}\text { taper and stop } \\
\text { clomipramine, } \\
\text { then start SNRI at } \\
\text { low dose§ }\end{array}$ \\
\hline moclobemide & $\begin{array}{l}\text { taper and stop } \\
\text { moclobemide, } \\
\text { then wait } \\
24 \text { hours for } \\
\text { washout before } \\
\text { starting above } \\
\text { drug }^{\S}\end{array}$ & $\begin{array}{l}\text { taper and stop } \\
\text { moclobemide, } \\
\text { then wait } \\
24 \text { hours } \\
\text { for washout } \\
\text { before starting } \\
\text { fluoxetine }\end{array}$ & $\begin{array}{l}\text { taper and stop } \\
\text { moclobemide, } \\
\text { then wait } \\
24 \text { hours } \\
\text { for washout } \\
\text { before starting } \\
\text { fluvoxamine }\end{array}$ & $\begin{array}{l}\text { taper and stop } \\
\text { moclobemide, } \\
\text { then wait } \\
24 \text { hours } \\
\text { for washout } \\
\text { before starting } \\
\text { vortioxetines }\end{array}$ & $\begin{array}{l}\text { taper } \\
\text { moclobemide, } \\
\text { start agomelatine }\end{array}$ & $\begin{array}{l}\text { taper and stop } \\
\text { moclobemide, } \\
\text { then wait } \\
24 \text { hours for } \\
\text { washout before } \\
\text { starting SNRI§ }\end{array}$ \\
\hline $\begin{array}{l}\text { phenelzine } \\
\text { tranylcypromine } \\
\text { (MAOIs) }\end{array}$ & $\begin{array}{l}\text { taper and stop } \\
\text { MAOI, then } \\
\text { wait } 14 \text { days for } \\
\text { washout before } \\
\text { starting above } \\
\text { drug }^{\S}\end{array}$ & $\begin{array}{l}\text { taper and stop } \\
\text { MAOI, then } \\
\text { wait } 14 \text { days } \\
\text { for washout } \\
\text { before starting } \\
\text { fluoxetine§ }\end{array}$ & $\begin{array}{l}\text { taper and stop } \\
\text { MAOI, then } \\
\text { wait } 14 \text { days } \\
\text { for washout } \\
\text { before starting } \\
\text { fluvoxamine§ }\end{array}$ & $\begin{array}{l}\text { taper and stop } \\
\text { MAOI, then } \\
\text { wait } 14 \text { days } \\
\text { for washout } \\
\text { before starting } \\
\text { vortioxetine }^{\S}\end{array}$ & $\begin{array}{l}\text { taper and stop } \\
\text { MAOI, start } \\
\text { agomelatine* }\end{array}$ & $\begin{array}{l}\text { taper and stop } \\
\text { MAOI, then } \\
\text { wait } 14 \text { days for } \\
\text { washout before } \\
\text { starting SNRI§ }\end{array}$ \\
\hline
\end{tabular}

Taper means gradual dose reduction, with lowering by increments every few days, usually over a period of 4 weeks, modified by patient experience, drug, illness and other factors.

All switches from one antidepressant to another may result in serious complications. Switches must be undertaken cautiously and under close observation.

The recommendations in this table are based on clinical experience, product information, empirical evidence and recommendations from other guidelines. It may be necessary to modify the switching process depending on patient, illness and interacting drug variables, determined by the patient's clinical progress. In appropriate circumstances expert prescribers may use less conservative switch strategies if justified by harm-benefit considerations arising from factors such as illness severity.

MAOI monoamine oxidase inhibitor

TCA tricyclic antidepressant
SNRI serotonin noradrenaline reuptake inhibitor

SSRI selective serotonin reuptake inhibitor

\section{An enlarged poster version of this \\ Switching-antidepressants table has been \\ inserted in the current issue of Australian \\ Prescriber. Extra copies are available \\ on request.}


mianserin mirtazapine

reboxetine

amitriptyline

imipramine

nortriptyline

doxepin

dothiepin

trimipramine

\section{(TCAs)}

taper drug, start above drug at low dose* taper drug, start reboxetine at $4 \mathrm{mg}$ * taper first drug, start

alternative TCA at

$25 \mathrm{mg}^{*}$

\section{clomipramine}

moclobemide

phenelzine

tranylcypromine

(MAOIs) taper clomipramine, then start above drug at low dose* taper clomipramine, then start reboxetine at $4 \mathrm{mg}^{*}$ taper clomipramine,

then start drug at

25 mg* taper and stop moclobemide, then wait 24 hours for washout before starting above drug $\S$

$\begin{array}{ll}\text { taper and stop } & \text { taper and stop } \\ \text { moclobemide, then } & \text { moclobemide, then } \\ \text { wait 24 hours for } & \text { wait 24 hours for } \\ \text { washout before } & \text { washout before } \\ \text { starting reboxetine }^{\S} & \text { starting above drug }\end{array}$

taper and stop MAOI, then wait 14 days for washout before starting reboxetine ${ }^{\S}$ taper and stop MAOI, then wait 14 days for washout before starting above drug $§$

taper and stop moclobemide, then wait 24 hours for washout before starting clomipramine ${ }^{\S}$

taper and stop MAOI, then wait 21 days for washout before starting clomipramine ${ }^{\S}$ clomipramine

\section{taper and stop clomipramine,} then wait 7 days for washout before starting moclobemide§

taper and stop drug, then wait 7 days for washout taper and stop drug, then wait 14 days (21 days for imipramine) before starting $\mathrm{MAOI}^{\S}$ starting above drug ${ }^{\S}$

\author{
taper and stop MAOI, \\ start moclobemide \\ while maintaining \\ MAOI dietary \\ restrictions for \\ 14 days $^{\S}$
}

\section{taper and stop clomipramine, then wait 21 days for washout before starting MAOI§}

taper and stop moclobemide, then wait 24 hours for washout before starting $\mathrm{MAOI}^{\S}$

taper and stop MAOI, wait 14 days for washout before starting other MAOI§

* A washout period of 2-5 half-lives (most frequently 2-5 days) between cessation of previous drug and the introduction of a new drug is the safest switching strategy from the point of view of drug interactions. In the indicated instances a washout period is not essential if switching is carried out cautiously and under close observation, and clinical considerations such as illness severity support harm-benefit considerations. Cautious cross taper (when the dose of the first drug is being reduced and the dose of the second drug is being increased at the same time so that the patient is taking both antidepressants) may be used in the indicated instances if appropriate and safe.

$+\quad$ Fluoxetine may still cause interactions 5 or 6 weeks after cessation (especially from higher doses) due to long half-life of drug and active metabolite.

$\ddagger$ Fluoxetine is likely to continue to elevate TCA concentrations for several weeks.

$\S$ Co-prescription of the two antidepressants in this instance is not recommended.

Adapted from references 2, 8-10 